\title{
An Epigram for Electromagnetic Field Theory Teaching Series by Professor Liang Changhong
}

\author{
Electromagnetic theory \\ develops explosively, \\ and covers \\ extensive contents \\ immense to \\ apply to \\ the universe, \\ subtle to \\ attend to \\ minute details. \\ Erudite scholar, \\ Professor Liang \\ has his \\ new work \\ for publication, \\ a frontier \\ science exploration. \\ An endless \\ learning process, \\ a great pleasure \\ to read it first.
}

Huang Hongjia

April 2012 
